\title{
L'arme non létale dans la stratégie militaire des Etats-Unis : imaginaire stratégique et genèse de l'armement
}

\section{Georges-Henri Bricet des Vallons}

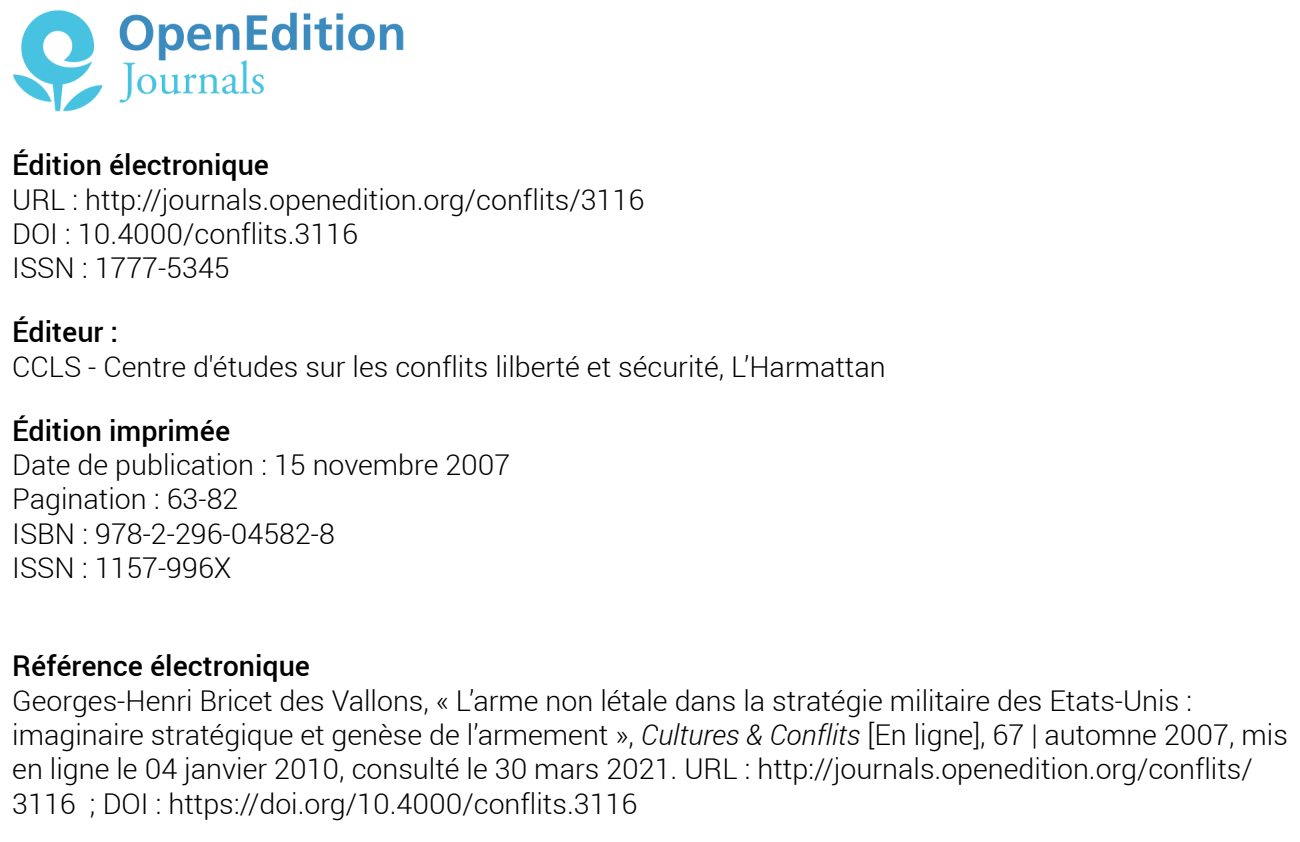




\title{
L'arme non létale dans la stratégie militaire des Etats-Unis : imaginaire stratégique et genèse de l'armement
}

\section{Georges-Henri BRICET DES VALLONS}

Georges-Henri Bricet des Vallons est doctorant en science politique, diplômé de l'Institut d'études politiques de Paris. Il est chargé de recherche à l'Institut de prospective et de sécurité en Europe (IPSE) et rédige actuellement une thèse sur les armes non létales.

\begin{abstract}
A pparue aux Etats-Unis dans les années 1960-1970, dans un contexte marA qué par l'émergence des masses contestataires et des mouvements de défense des droits civiques, la théorie de la non-létalité a gagné à partir du début des années 1990 une place centrale dans la réflexion militaire sur les conflits asymétriques et la guerre urbaine. La mise en service à titre d'expérimentation en 2006 en Irak, dans le cadre de la politique de contre-insurrection, d'armes comme le Long Range Acoustic Device (LRAD) et l'Active Denial System (ADS) a signé une étape primordiale dans le développement de systèmes antipersonnels de nouvelle génération. L'apparition de ces armes à énergie dirigée amène à s'interroger sur la nature de la révolution scientifique et stratégique que tente de promouvoir la théorie de la non-létalité.
\end{abstract}

Nous verrons que celle-ci se rattache à plusieurs égards à une transformation d'ordre «biopolitique » de l'action militaire. Pour Foucault, l'objet même du biopolitique est la population, «la multiplicité des hommes» considérée comme une "masse globale affectée de processus d'ensemble qui sont propres à la vie ${ }^{1}$ ». Le biopolitique est à entendre comme un processus complexe de gestion/régulation des corps (corps-machine) et des populations (corps-espèce) en tant qu'objets de pouvoir :

1. Foucault M., Il faut défendre la société, Cours au Collège de France (1975-1976), Paris, Gallimard/Seuil, 1997, p. 216. 
«Les disciplines du corps et les régulations de la population constituent les deux pôles autour desquels s'est déployée l'organisation du pouvoir sur la vie. La mise en place [...] de cette grande technologie à double face - anatomique et biologique, individualisante et spécifiante, tournée vers les performances du corps et regardant vers les processus de la vie - caractérise un pouvoir dont la plus haute fonction désormais n'est peut-être plus de tuer mais d'investir la vie de part en part. [...] La vieille puissance de la mort où se symbolisait le pouvoir souverain est maintenant recouverte soigneusement par l'administration des corps et la gestion calculatrice de la vie 2 ».

Dans la mesure où le concept de non-létalité postule pour finalité fonctionnelle d'un armement spécifique l'évitement de la mort et/ou la sauvegarde de la vie, il est manifestement «biopolitique ». Le concept d'arme non létale s'inscrit dans ce recouvrement historique d'un thanato-pouvoir centralisé et restreint (société disciplinaire) par un biopouvoir massifiant et diffus (société de contrôle).

Partant de ce point de vue, cette analyse a pour objectif de décrypter le processus de légitimation dont le concept de non-létalité a fait l'objet dans le discours politico-militaire américain et son inscription dans la théorie stratégique occidentale. Mettre en relief le rôle joué par l'imaginaire stratégique et la « fiction technologique » - c'est-à-dire la science « prospective »-, dans la genèse technique d'un armement, est capital pour saisir l'émergence d'une doctrine militaire. Il s'agit ici de comprendre comment une projection discursive - la théorie de la non-létalité - incluse dans une mythologie stratégique - la « Transformation » a opéré et continue d'opérer dans la conception et la production d'un système d'armes ; comment donc la formation du discours stratégique influence, informe et instruit l'évolution des pratiques de la recherche techno-scientifique et ses fins.

\section{L’arme non létale : généalogie politique, genèse doctrinale}

Il est difficile d'identifier un acteur institutionnel prépondérant dans le développement des armes non létales (ANL), tant les recherches ont reposé dès le départ sur une base hybride, à la fois civile et militaire. Trois acteurs étatiques ont néanmoins joué un rôle de premier plan dans l'institutionnalisation des programmes de recherche : les départements de la Justice, de l'Energie et de la Défense.

\section{Origines civiles}

Sur le plan civil, c'est le National Institute of Justice (NIJ), organe de recherche du département de la Justice, qui, depuis la fin des années 1980,

2. Foucault M., Histoire de la sexualité 1 : la volonté de savoir, Paris, Tél/Gallimard, 1994, pp. 183-184. 
finance le plus activement, en concertation avec l'ARDEC ${ }^{3}$, le développement d'armes incapacitantes destinées au contrôle des populations civiles, carcérales notamment. En 1992, le NIJ demande l'aide technique du département de l'Energie pour mettre sur pied un programme de technologies spéciales ${ }^{4}$. Le service de renseignement de l'Energie étudiait à l'époque des applications pouvant protéger efficacement l'abord des sites nucléaires du pays ${ }^{5}$. En 1993, les financements du NIJ s'orientent vers des projets mettant en jeu les stimuli du corps humain. Un rapport du NIJ décrit ainsi le sens des recherches menées :

«Le programme impose des recherches croissantes sur [...] des technologies variées pouvant produire des réactions physiologiques temporaires, comme la nausée, le vertige et la désorientation. Fait l'objet d'étude la sensibilité du corps humain au son, à la lumière, aux ondes électromagnétiques ionisantes ou non ionisantes. Le but du projet est de comprendre à quoi réagit le corps humain et de développer un procédé, un outil ou une arme qui puisse reproduire et provoquer cette réaction. Ces armes pourraient neutraliser de façon temporaire un individu ou un groupe sans lésion physiologique permanente 6 ».

Les programmes du NIJ vont naturellement éveiller l'intérêt des institutions policières, notamment celui de l'American Correctionnal Association et de la National Association of Sheriffs, intensifiant ainsi la recherche prospective sur l'amélioration des techniques de maintien de l'ordre (lawe enforcement) qui conduira à la mise en service du Taser ${ }^{7}$ et, dans certains Etats, à l'emploi de ceintures de contention électriques (REACT Belt $\left.{ }^{8}\right)$ dans les pénitenciers.

Les recherches vont s'accélérer sous l'administration Clinton. En 1993, suite notamment aux émeutes de Los Angeles en 1992 et à la tragédie du siège de Waco, Janet Reno, secrétaire d'Etat à la Justice, suggère à la Défense et à la CIA la mise en place d'une collaboration institutionnelle visant à développer des technologies duales pouvant s'appliquer autant aux domaines civils que militaires. Cette démarche est ratifiée par un mémorandum d'accord signé en avril 1994 entre le département de la Justice et celui de la Défense, sur le par-

3. ARDEC : Armament Research, Development and Engineering Center.

4. Davidson N., The Development of "Non-Lethal" Weapons duringt the 1990's, occasional paper $\mathrm{n}^{\circ} 2$, rapport du projet de recherche sur les armes non létales, université de Bradford, mars 2007, pp. 2-8.

5. Ibid.

6. Seaskate Inc., The Evolution and Development of Police Technology, Washington DC, National Institute of Justice, 1998, p. 46. Notre traduction.

7. Le Taser, du nom de son principal fabriquant, est une arme à impulsions électriques en dotation dans les forces de police occidentales depuis une dizaine d'années.

8. REACT Belt: Remote Electronically Activated Control Technology Belt. Cette ceinture de contention électrique, développée par Stun Tech (aujourd'hui Stinger Systems), introduite dans les prisons de seize Etats américains au début des années 1990, émet pendant huit secondes une décharge de cinquante volts dans les reins du prisonnier. 
tage de technologies susceptibles de renforcer les opérations non directement guerrières (Operations other than War) et les opérations de maintien de l'ordre 9 . Le programme, supervisé par la DARPA, regroupe des membres du NIJ, du FBI, du Bureau of Prisons et de l'US Army et définit alors sept domaines technologies prioritaires, au titre desquels figurent les armes non létales.

La théorie de la non-létalité n'a rien, à l'époque, de révolutionnaire. Elle a déjà connu un développement continu depuis les années 1960, notamment dans le cadre d'études portant sur l'amélioration des technologies cinétiques (balles de caoutchouc), chimiques (gaz au poivre) et électriques (pistolets assourdissants). Ainsi que le notent Lewer et Schofield ${ }^{10}$, dans le contexte historique de la Guerre froide, la polarisation des études stratégiques sur les armes de destruction massive et l'Air Land Battle ne pouvait entraîner qu'un intérêt mineur pour les armes non létales de la part du corps militaire.

Il existe cependant à l'époque une nette séparation conceptuelle entre développement civil et militaire du concept. Les études émanant du monde civil mirent davantage l'accent sur le potentiel d'extension du contrôle social qu'offrait le non-létal et eurent tendance à explorer ses aspects antipersonnel - thématique du «contrôle des foules » qui imprégnera alors par contrecoup et en profondeur la doctrine de maintien de la paix des armées occidentales 11 - alors que les organismes militaires perçurent et évoquèrent en premier lieu le concept en termes de gains stratégique et capacitaire, portant plus naturellement l'accent sur les armes antimatériel. Les références au potentiel militaire de la non-létalité restent cependant relativement marginales. A cet égard, l'étude de Joseph Coates publiée en 1970 par l'Institute for Defense Analyses 12 constitue une exception notable : elle élargit considérablement la portée du concept et défend son emploi dans le cadre des guerres limitées et des conflits de basse intensité. Coates fait preuve d'une acuité prospective certaine en discernant une des évolutions majeures du contexte conflictuel mondial : «Combattants et civils seront de plus en plus entremêlés et la dilution de cette distinction ira croissante dans la plupart des conflits à venir. Ce sera particulièrement le cas du combat en zone urbanisée ».

Intervenant à la charnière des années 1980-1990 et de l'effondrement politico-militaire du bloc soviétique, l'effet de rupture est essentiellement technologique, les recherches sur des domaines prospectifs comme l'énergie dirigée

9. Department of Defense, Department of Justice, Memorandum of Understanding between Department of Defense and Department Of Justice on Operations other than War and Law Enforcement, 20 avril 1994.

10. Lewer N., Schofield S., Non-Lethal Weapons. A Fatal Attraction? Military Strategies and Technologies for $21^{\text {st }}$ Century Conflict, Londres, éd. Zed Books, pp. 34-35, 1997. Notre traduction.

11. Voir Potier E., «Imaginaire du contrôle des foules dans l'armée de terre française », Cultures \& Conflits, $\mathrm{n}^{\circ} 56,2004$, pp. 35-49.

12. Coates J., Nonlethal and Nondestructive Combat in Cities Overseas, Washington DC, Institute for Defense Analyses, Science \& Technology Division, 1970, p. 1. Notre traduction. 
laissant entrevoir pour la première fois des applications industrielles. Il s'agit, dans le cadre de la RAM (Révolution dans les affaires militaires), de développer une panoplie d'armes tous azimuts ayant pour objectif une paralysie « globale » de l'ennemi, en circonscrivant et en mobilisant toutes les possibilités offertes par la prospective technologique : lasers, ondes acoustiques, électromagnétiques, matériaux super-adhésifs, super-caustiques, etc. Le spectre des virtualités industrielles des ANL est si large qu'il concerne toutes les filières technoscientifiques imaginables et offre donc au complexe militaro-industriel un marché particulièrement lucratif - dynamique économique que viendra confirmer l'essor commercial du Taser à l'échelle mondiale.

\section{Fondements militaires}

C'est en 1990, sous l'impulsion majeure du Strategy Global Council, thinktank conservateur alors dirigé par Ray Cline, ex-directeur de la CIA, que la question du non-létal acquiert véritablement une place centrale dans le débat stratégique américain. Née de la collaboration entre des «futurologues » (Alvin et Heidi Töffler), des écrivains de science-fiction (Chris et Janet Morris), et le colonel John Alexander, la réflexion sur une nouvelle génération d'armes incapacitantes va connaître une légitimation politique avec l'échec américain en Somalie. Le traumatisme de l'opération United Shield 13 finira d'établir, aux yeux des décideurs politiques et militaires, la nécessité, dans un contexte polémologique marqué par une urbanisation croissante du champ de bataille et par une policiarisation de l'action militaire, de combler le vide capacitaire sur l'échelle des règles d'engagement entre réponse minimale et réponse létale. Le Concept for NonLethal Capabilities in Army Operations de décembre 1996 souligne ainsi que :

«Le contrôle des foules dans la conduite des missions de maintien de la paix et d'assistance humanitaire est devenu pour l'armée une tâche aussi courante que la destruction des blindés ou de l'artillerie ennemis en temps de guerre 14 ».

Un rapport du Center for Strategic and International Studies (CSIS) think tank au sein duquel avait germé l’idée de RAM - datant de 1993, souscrivait en ces termes au « potentiel révolutionnaire » des ANL :

«Si les forces américaines sont capables, à travers l'électronique, l'électromagnétique, l'énergie dirigée ou d'autres moyens, de neu-

13. Comme le souligne Dennis Herbert, l'intérêt des marines pour les ANL à l'occasion du conflit somalien a joué un rôle de catalyseur : "l'enthousiasme $d u$ Marines Corps pour les armes non létales a eu une influence positive sur les autres forces armées ", in Herbert D., "Non-lethal weaponry: from tactical to strategic applications", Joint Force Quaterly, printemps 1999.

14. Concept for Non-Lethal Capabilities in Army Operations, pamphlet 525-73, United States Army Training and Doctrine Command (TRADOC), décembre 1996. Notre traduction. 
traliser ou d'asphyxier les forces ennemies sans les détruire ou les tuer, la conduite des opérations militaires en serait révolutionnée. Le calcul global des coûts, des bénéfices et des risques changerait alors et pour les Etats-Unis et pour leurs adversaires 15 »

L'avantage d'un tel système d'arme est évident : d'un point de vue politicomilitaire, il fournit un argument pour légitimer des opérations qui n'auraient pu l'être avec des armes conventionnelles, tandis que d'un point de vue tactique, il offre aux décideurs sur le terrain une option supplémentaire d'intervention.

Parmi ces personnages fondateurs, John Alexander est aujourd'hui connu comme la figure initiatique de la théorie de la non-létalité. Ce colonel d'infanterie de l'US Army, impliqué à l'époque du Vietnam dans le programme «Phoenix », chef de projet sur les applications militaires de l'énergie dirigée au laboratoire de Los Alamos dans les années 1980 et retraité en 1988, va bâtir à travers une série d'articles 16 le cadre de la doctrine d'emploi américaine. Alexander s'appuie sur le non-létal pour élaborer une doctrine de la paralysie stratégique - dite aussi de la « camisole »-, fondée sur une panoplie d'armes anti-infrastructure et anticapacité. On peut considérer que cette doctrine a partiellement été mise en pratique lors du conflit kosovar ${ }^{17}$ avec l'emploi massif de bombes au graphite pour neutraliser les réseaux électriques serbes et lors de l'invasion de l'Irak en avril 2003.

\section{Concept et doctrine}

Dans le sillon conceptuel tracé par le Global Strategy Council, naît en mars 1991, sur recommandation du sous-secrétaire d'Etat à la Défense, Paul Wolfowitz, qui en prendra alors la tête, un groupe de réflexion informel, le Non-Lethal Strategy Group (NSG). William Perry, secrétaire à la Défense sous Clinton, en perpétuera l'existence. Cette cellule de coordination a pour but initial de poursuivre en toute discrétion les travaux sur les gaz incapacitants (au moment où l'administration américaine était sur le point de signer la Convention sur l'interdiction des armes chimiques), mais elle va étendre rapidement ses acti-

15. Mazarr M., The Military Technical Revolution: a Structural Framework, Washington DC, CSIS, p. 43, 1993. On pourra également se référer au rapport du CFR sur la question : NonLethal Technologies: Military Operations and Implications, New-York, Council on Foreign Relations Press, 1995. Notre traduction.

16. Se référer pour l'essentiel à : Alexander J.B., Future War: Non-Lethal Weapons in Twenty First Century Warfare, New-York, St-Martin Press, 1999; Alexander J.B., Winning the War: Advanced Weapons, Strategies, and Concepts for the Post-9/11 World, New-York, St-Martin Press, 2003 ; “Antimateriel Technology”, Military Review, vol. 69, n¹0, pp. 29-41, octobre 1989; "Non-Lethal weapons to gain relevancy in future conflicts”, National Defense, mars 2002 ; Alexander J.B., "Optional lethality: evolving attitudes toward nonlethal weaponry", Harvard International Review, été 2001, pp. 64-69.

17. C’est aussi pendant le conflit du Kosovo qu'ont été employées pour la première fois dans le cadre d'une opération extérieure multinationale des grenades éponges et autres munitions cinétiques pour le contrôle des foules. Voir: "Not your average weapon”, Stars E Stripes, dimanche 9 avril 2000. 
vités à tous les domaines exploratoires du non-létal. Le NSG assure dès lors un travail de formalisation doctrinale qui débouche en juillet 1996 sur la Directive 3000.3 , document fondateur de la théorie de la non-létalité ${ }^{18}$. On y trouve la définition matricielle du concept :

«Les armes non létales sont des armes discriminantes qui sont explicitement mises au point et principalement utilisées pour frapper d'incapacité le personnel et le matériel, avec un minimum de risque mortel, de lésions permanentes au personnel et de dommages indésirables aux biens et à l'environnement ».

Le débat entre détracteurs et partisans du concept a dès lors porté sur la validité de la distinction conceptuelle entre armes létales et armes non létales. Les multiples acceptions du concept exprimaient alors son flou - non létal, moins létal, pré-létal, semi-létal, sublétal, à létalité réduite - comme le trop grand nombre de ses fonctions et de ses virtualités d'application. Cependant, la définition donnée par la doctrine d'emploi révèle ce qui est une fausse ambiguïté, à savoir que le caractère non létal, réversible, discriminant et proportionnel de ces armes n'est pas garanti dans tous les scénarios d'emplois possibles, comme l'explique la politique de l'OTAN en la matière, calquée sur la définition américaine : "Les armes non létales n'offrent pas nécessairement une probabilité nulle de provoquer des pertes en vies humaines ou des lésions permanentes 19 ».

De la même façon, la dotation en armes non létales ne saurait constituer une obligation d'emploi :

«L'existence, la présence ou l'effet potentiel des armes non létales ne constitue pas une obligation d'emploi de ces armes ni n'impose une norme supérieure, ou des restrictions supplémentaires, au recours à la force létale. La force conserve dans tous les cas la possibilité d'un usage immédiat des armes létales conformément au droit national et international en vigueur et aux règles d'engagement agréées 20 ».

La relativité du concept est donc admise comme flagrante. Par un glissement lexical le non-létal est avant tout à entendre comme less than lethal, " moins que létal ». Il apparaît que le débat sur la distinction conceptuelle entre létal et nonlétal tient d'un certain pharisianisme ou d'une naïveté coupable de la part de ses détracteurs comme de la part de ses partisans ${ }^{21}$, la doctrine militaire américaine

18. DoD Directive 3000.3, Policy for Non-Lethal Weapons, datant du 9 juillet 1996, certifiée le 21 novembre 2003. Notre traduction.

19. Politique de l'OTAN sur les armes non létales du 13 octobre 1999, chiffre III, titre 8.

20. Ibid., titre 7.

21. C'est la première fois que l'invention d'une arme repose, dans sa justification rhétorique, sur un fondement pseudo-humanitaire et on notera avec intérêt l'insistance de nombre de décideurs politico-militaires américains sur la supériorité éthique que sont censées conférer ces 
n'ayant à aucun moment nourri l'illusion d'une « guerre sans morts » ou d'une « létalité zéro ». C’est sur ce point d'interprétation qu'Alexander assied le concept et met en garde contre une vision irénique et essentialiste de la non-létalité : en effet, si «les armes non létales antipersonnel sont destinées à un usage de la force qui protège et sauvegarde la vie », celui-ci note "qu'aucune arme, peu importe sa bénignité, n'est totalement non létale» 22 . De même que la prise en compte de l'asymétrie comme paradigme conflictuel dominant ne saurait marginaliser ou invalider la pensée militaire de l'affrontement symétrique et conventionnel, le non-létal ne disqualifie en aucun cas la pertinence de la force létale, mais opère comme un «multiplicateur de force ». Selon les circonstances d'emploi et la physiologie de la cible, toute ANL est potentiellement mortelle. Le débat sur une acception stricte de l'innocuité du non-létal paraît donc quelque peu artificiel. Il conduira à l'adoption et à la préférence du terme d' " armes à létalité réduite ", moins contraignant sur le plan éthique, et qui permet de ménager une marge d'appréciation et d'erreur au plan juridique. Au souci de vérifier dans le temps la garantie technique des effets non létaux, répondra en 2000 la mise en place par la Joint Non-Lethal Weapons Directorate (JNLWD) d'une cellule d'experts, l'Human Effects Advisory Panel, chargée d'évaluer l'impact et la prévisibilité des effets biologiques des systèmes sur le corps humain.

En 1998, la doctrine de la non-létalité connaît sa première formulation officielle dans le Joint Concept for Non-Lethal Weapons ${ }^{23}$. Celui-ci marque une mise à distance, voire un recul, de la doctrine vis-à-vis de la vision stratégique épousée par le Global Strategy Council. Le concept interarmées, dans un effort d'épuration, exclut du champ d'application du non-létal les domaines de la guerre psychologique et de la guerre électronique. Il se découpe en deux registres principaux : les armes antipersonnel et les armes antimatériel ; cette catégorie étant à la racine de deux sous-registres, les armes anti-infrastructure et les armes anticapacité (ou anti-système d'arme). Ces deux registres disparates - armes antipersonnel et armes antimatériel - constituent aujourd'hui le cœur de la doctrine de la non-létalité ${ }^{24}$.

\section{Acteurs et réseaux}

Suite à la publication de la Directive 3000.3 en 1996, le Congrès américain autorise le développement d'armes non létales et officialise l'existence du NSG

armes à la force qui les emploie, idée caractérisée par la récurrence dans les écrits militants de la notion de "moral preeminence». Voir à ce sujet : Brown J.B., "Non-lethal weapons and moral preeminence in peacekeeping operations", Naval War College, 2005.

22 . Alexander J.B., "Optional lethality: evolving attitudes toward nonlethal weaponry", Harvard International Review, été 2001, pp. 64-69. Notre traduction.

23. Joint Concept for Non-Lethal Weapons, commandant du Marine Corps, 5 janvier 1998. Notre traduction.

24. On notera le caractère problématique de cette articulation. C'est en effet un non-sens que d'appliquer une notion biologique - la létalité - aux armes antimatériel, qui devrait alors constituer une doctrine propre. 
sous la forme du JNLWD. Evoluant au sein de la division Special Operations and Low-intensity Conflict du département de la Défense, ce comité directeur est doté d'un budget annuel d'environ 30 millions de dollars ${ }^{25}$. Le JNLWD, établie sur la base de Quantico en Virginie, devient pleinement opérationnelle en janvier 1997, avec le Joint Service Memorandum of Agreement ${ }^{26}$ qui en établit la structure hiérarchique et l'organigramme interagences. Le Marines Corps est désigné comme l'agent exécutif du programme intégré et le coordinateur central des projets de développement. Il prend en charge le volet de la recherche opérationnelle, en liaison avec les acteurs scientifiques suivants : les entités dépendantes de la Défense, l'ARDEC 27, la DARPA, l'ANSEC 28 (US Army), les laboratoires de recherche appliquée de Los Alamos et de Kirtland (US Air Force) ; les laboratoires nationaux de Lawrence Livermore en Californie et de Sandia à Albuquerque supervisés par le département de l'Energie; on peut également citer les centres de recherche d'Etat rattachés aux armées tels que l'Institute for Non-Lethal Defense Technologies de l'université de Pennsylvanie et le Non-Lethal Technology Innovation Center de l'université du New Hampshire. Los Alamos est essentiellement connu pour être à la pointe des recherches sur les armes à énergie dirigée, lasers tactiques de haute puissance et canons à ondes millimétriques de basse puissance. Sandia assure les travaux sur les mousses paralysantes et les hyper-caustiques, tandis que Lawrence Livermore poursuit, officiellement jusqu'en 1997, les recherches sur les armes chimiques incapacitantes. Etant donné la perméabilité civil-militaire, étatique-privé, propre aux réseaux militaro-industriels américains, chaque programme de recherche fait évidemment l'objet de multiples partenariats avec l'industrie de défense: Boeing (Advanced Tactical Laser), Raytheon (Active Denial System), Primex aerospace et tasertron Co. (mines antipersonnel électriques et munitions chimiques), Mission Research Corp (Pulsed Energy Projectile), entre autres, sont parties prenantes de ses programmes depuis plusieurs années.

En hybridant maintien de l'ordre et maintien de la paix, le non-létal apparaît comme un exemple inédit de concept stratégique «global », échafaudé sur une base «interagences » et à finalité « duale », chevauchant la frontière entre civil et militaire. C'est en effet la première fois qu'un armement, dans sa conception, n'a pas pour finalité opérationnelle de donner la mort, mais au contraire de l'éviter, voire de sauvegarder la vie. Le non-létal apparaît donc bien comme un concept « biopolitique » 29. Par «biopolitique », nous entendons ici spécifiquement le déploiement global - local, national et international - et l'autonomisa-

25. Le budget de la JNLWD connait, à la partir de la moitié des années 1990, une augmentation constante : de 9,3 millions de dollars en 1997, il passe à 16,1 millions en 1998 et à 33,9 millions en 1999, pour atteindre 43 millions en 2004.

26. Joint Memorandum of Agreement, Office of the Secretary of Defense, datant du 22 mars 1996, révisé le 23 juin 1999. Notre traduction.

27 . Voir note 3.

28. ANSEC : Army Nonlethal Scalable Effects Center.

29. Cette marque biopolitique du concept est prégnante dans la devise de la JNLWD : «Pax custimus, vita custimus », « Gardons la paix, gardons la vie ». 
tion croissante des modalités et des techniques de régulation et de contrôle - étatiques, paraétatiques et privées - destinées à gérer la vie dans sa dimension économique et sécuritaire. En effet, le biopolitique en tant que processus de perpétuation de l'espèce, par définition amoral, ne cesse de promouvoir et d' « installer des mécanismes de sécurité autour de [l']aléatoire inhérent à une population d'êtres vivants 30 ", de propager des modes de régulation et des outils de gestion visant à circonscrire les fluctuations du vivant. De ce point de vue, l'arme non létale capte une transformation majeure de l'imaginaire politique et militaire de la «technologie $d u$ corps $^{31}$ » et se pose comme un mythe stratégico-scientifique - «neutraliser sans détruire », «vaincre sans tuer », ou plutôt « vaincre en laissant vivre »; mythe qui, nous allons le voir, participe lui-même d'une mutation extensive - d'une «globalisation » $-\mathrm{du}$ contrôle socio-militaire.

\section{La non-létalité dans la mythologie de la «Transformation »}

La compréhension du processus de légitimation dont a fait l'objet la théorie de la non-létalité au sein de la pensée stratégique américaine ne saurait se satisfaire d'une lecture simplement et exclusivement événementielle ou factorielle. Toute révolution scientifique fonctionne comme une mutation épistémologique. Pour appréhender l'émergence du non-létal dans la théorie stratégique et sa promulgation comme micro-révolution, technologique et militaire, il importe de le replacer dans la perspective plus fondamentale de la «Transformation ", car à bien des égards, cette légitimation a été le résultat à la fois d'une mutation de la culture stratégique américaine et de l'apparition d'anomalies et de discontinuités dans la nature même des conflits que le concept de non-létalité a tenté de résorber.

\section{L'arme non létale comme "paradigme » techno-militaire et « mythe » doctrinal}

Si l'on se rapporte à la définition de la RAM - aujourd'hui requalifiée sous le registre de la «Transformation »- généralement attribuée à son concepteur, Andrew Marshall :

«Une révolution dans les affaires militaires est un changement majeur dans la nature de la guerre suite à l'application de nouvelles technologies innovantes qui, combinées à des changements radicaux dans les doctrines militaires et opérationnelles, ainsi que dans les concepts organisationnels, altère fondamentalement le caractère et la conduite des opérations militaires 32 ».

30. Foucault M., Il faut défendre la société, op. cit., p. 219.

31. Foucault M., Surveiller et punirs: naissance de la prison, Paris, Gallimard/Seuil, 1975, p. 34.

32. Citation traduite par C. Wasinski, rapportée dans McKitrick J., Blackwell J., "The Revolution in military affairs", in Schneider B.R., Grinter L.E., (dir.), Battlefield of the Future. $21^{\text {st }}$ Century Warfare Issues, Air War College, septembre 1995. Voir sur le sujet: Wasinski C., "Créer une révolution dans les affaires militaires: mode d'emploi », Cultures E Conflits, $\mathrm{n}^{\circ} 64,2006$. 
Le changement ici décrit est assez similaire au processus de transformation du « paradigme » scientifique décrit par Thomas Kuhn dans La Structure des révolutions scientifiques ${ }^{33}$, " paradigme » qui peut fournir un procédé d'analogie cohérent pour traduire les mutations du discours stratégique américain. Selon Kuhn, toute révolution scientifique est fondée sur l'avènement d'un paradigme, d'un discours qui fonctionne, au sein d'une communauté doctrinale, comme une idéologie de la vérité scientifique. En somme, tout paradigme est un «mythe fondateur » : en tant que «modèle » et « heuristique », il constitue un «mythe», un récit idéologique et fournit une «fondation» herméneutique à un discours donné, en ce qui nous concerne la RAM et ce que nous identifions comme un de ses principaux symptômes, la théorie de la non-létalité. C'est par l'avènement d'un paradigme à la place d'un autre, frappé de "crise », que s'opère une "révolution » scientifique. « Crise » n'est pas à entendre ici comme une rupture résultant d'un processus cumulatif, mais réfère à l'apparition d'anomalies qui bouleversent ou opacifient l'interprétation d'un phénomène donné. L'émergence de ces «anomalies » provoque, au sein de la communauté doctrinale, la prise de conscience que le paradigme existant a cessé d'opérer dans l'exploration de certains domaines et qu'un nouvel outil théorique est nécessaire pour appréhender ce régime d'anomalies. C'est à ce moment que la «science normale » se transforme en «science extraordinaire »-période qui passe le plus souvent inaperçue du plus grand nombre - et que celle-ci, une fois le nouveau paradigme intégré et mis en forme dans un discours théorique, rebascule à nouveau dans sa période dite «normale ». Ce basculement ne signifie donc pas automatiquement l'obsolescence ou l'invalidation du paradigme en «crise ». Des paradigmes concurrents peuvent coexister durablement, coexistence qui est à l'origine du « débat » scientifico-stratégique. De même, plusieurs paradigmes distincts, voire partiellement contradictoires, peuvent cohabiter au sein d'une même doctrine et se nourrir l'un l'autre sans menacer la légitimité et la structure de l'édifice théorique.

Le cas de la RAM, ni véritable méthode, ni doctrine, est en ce sens exemplaire. Nébuleuse, elle opère ainsi dans le débat stratégique américain comme une tentative de formalisation englobante de toutes les évolutions stratégiques et tactiques permises par le déploiement global des techniques de guerre et de contrôle, civiles et militaires. La concurrence des projections stratégiques, des doctrines militaires, des légendes technologiques, dont les think tank sont les matrices et les caisses de résonance, revêt alors une importance décisive dans la conception et la production de l'armement. Cette dimension psychologique et idéologique du «paradigme stratégique » régit la légitimation de la recherche techno-militaire.

\section{Globalisation stratégique : armes non létales et continuum de force}

Si l'on applique l'analyse des déterminants paradigmatiques au concept de non-létalité, on dégagera aisément les inflexions polémologiques propres au

33. Kuhn T., La Structure des révolutions scientifiques, Paris, Belin, 1962. 
concept : à la « destruction » de l'ennemi comme principe de la guerre, il substitue sa «paralysie »; l'impératif de «contrôle du milieu » supplante celui de la « conquête du territoire »; enfin il conduit à une policiarisation de l'action militaire et à une militarisation de l'action policière. Surtout en tant que concept présupposé « révolutionnaire », l’arme non létale fournit une assise théorique à l'hypothèse du continuum de force.

Ce basculement de paradigme finira de marginaliser les schémas qui organisaient la praxéologie de la stratégie moderne fondée sur l'affrontement symétrique ou conventionnel dans le contexte de la dissuasion nucléaire. De la guerre du Golfe à l'actuelle guerre d'Irak, en passant par la Somalie, le Liberia, Haïti, les Balkans, le Kosovo, puis l'Afghanistan, il finira de marginaliser la pertinence de la vision stato-centrique clausewitzienne ${ }^{34}$ : ami/ennemi, sécurité extérieure/sécurité intérieure, civil/militaire. Pour citer Lucien Poirier : "Clausewitz, indépassable dans le discours de la guerre, devait être néanmoins dépassé dans le déploiement d'un discours de la stratégie dont la guerre n'est plus qu'une phase dans le spectre des états de conflit 35 ». Une telle évolution peut être caractérisée, pour reprendre une expression d'Alain Joxe ${ }^{36}$, comme une " globalisation stratégique» de la guerre. Présence permanente du média dans le conflit, multiplication des conflits urbains prolongés (Palestine, Liban, Somalie, Côte d'Ivoire, Irak, Afghanistan), dilution de la distinction sémiologique entre militaire et civil, combattants et non-combattants, militaires et paramilitaires (sociétés militaires privées)... S’il y a une matrice commune à toutes ces évolutions conflictuelles, c'est bien celle de la guerre donnée comme système de contrôle transversal et continuum de force. De ce constat naîtront les concepts de « réversibilité » et de « graduabilité » subsumés dans la théorie de la « guerre des trois blocs » 37 qui structure depuis une quinzaine d'années la pensée tactique du combat urbain. Les principes du non-létal se déduisent donc des mêmes postulats qui sous-tendent la théorie du continuum de force : graduabilité, réversibilité, modularité. Ce sont autant de choix stratégiques, autant d'options tactiques qui se subordonnent à une mutation extensive du contrôle socio-militaire. Transversalité de l'action, réversibilité du mode opératoire, graduabilité de la réponse armée. Dans ce sillon, l'idéologie du « zéro mort », à laquelle est liée symboliquement le non-létal, fera sienne, non plus l'illusion judéo-chrétienne de la guerre «juste », mais l'illusion biopolitique de la guerre «propre».

34. Pour une analyse de la réception de Clausewitz dans l'armée américaine voir : Colson B., « La stratégie américaine de sécurité et la critique de Clausewitz », Stratégique, n76, 1999-4.

35 . Poirier L., Les Voix de la stratégie. Généalogie de la stratégie militaire, Guibert, Jomini, Paris, Fayard, 1985, pp. 315-484.

36 . Joxe A., «La globalisation stratégique », Cahier d'études stratégiques, n40-41, 2006.

37. Le concept de «Three Block War» a été développé par le général Charles Krulak pour décrire la complexité du spectre opérationnel de la guerre en zone urbanisée, qu'il découpe en trois phases d'intensité (basse/moyenne/haute). Voir Krulak C., “The strategic corporal: leadership in the Three Block War”, Marines Magazine, Air University, 1999. 
Produit par excellence de la Military Technical Revolution(MTR), le concept de non-létal se situe à la croisée de deux courants doctrinaux - de deux idéologies de la guerre projetée comme futur permanent - qui structurent aujourd'hui la pensée stratégique de la RAM : la «guerre-réseau-centrée » et la "guerre de quatrième génération ». L'une et l'autre ne constituent au final que deux versants complémentaires de la RAM : la «guerre-réseau-centrée » («Network Centric Warfare») assure le volet de la transformation technologique de l'appareil de guerre américain, tandis que la « guerre de quatrième génération » ( $4^{\text {th }}$ Generation War », 4GW) défend une révolution structurelle des affaires stratégiques et de la direction politique de la guerre 38 liée à la prise en compte de l'asymétrie comme modèle stratégique dominant des futurs conflits. Ces deux théories constitutives de la RAM partagent un dénominateur idéologique commun, celui de la guerre comme "système des systèmes ", comme continuum médiatique, technologique, diplomatique et militaire. Cette évolution vers un « méta-système » met en relief un certain nombre de structures fondamentales de la pensée de la "guerre globale » ou, dans sa qualification stratégique américaine, de la « force objective » 39 : omniscience, omnipotence, omniprésence du contrôle socio-militaire. Dans cette "vision », le système de guerre américain, soustrait aux contingences géopolitiques et aux servitudes du droit international de la guerre, vise un équilibre astatique - indifférent dans toutes les positions. Il s'agit pour l'armée américaine non pas de faire des opérations de maintien de la paix ou de la gestion post-conflit l'alpha et l'omega de son action ${ }^{40}$, mais de répondre à l'injonction de la Full Spectrum Dominance que vise le modèle de la RAM à travers la Joint Vision 2010 et 2020, c'est-à-dire à transformer le spectre des opérations guerrières - le Three Block War - en un continuum adapté aussi bien à des opérations de haute intensité qu'à des interventions de maintien de la paix. Il ne faut cependant pas surdéterminer le rôle joué par cette idéologie du "méta-système » dans la production du discours stratégique. La doctrine américaine elle-même ne se laisse leurrer que partiellement par cette chimère. Comme l'explique l'amiral Owens, père de la NCW (Network Centric Warfare):

«Un système de systèmes n'offre pas l'omniscience ni l'omnipotence. [...] Ce qui compte pendant une guerre, c'est l'influence relative de ce que certains ont appelé la brume et la friction du conflit

38. La théorie de la « guerre de quatrième génération » est à l'origine directe ou indirecte de nombreux concepts en vogue comme le DIME (Diplomatic, Information, Military, Economic), les $\mathrm{EBO}$ (Effects Based Operations), les opérations interagences (Interagency Operations) ou encore les opérations informationnelles (Information Operations). Pour approfondir ces concepts, voir : Bricet des Vallons G.-H., «L'intégration interagences : révolution dans les affaires civilo-militaires ? ", Défense et sécurité internationale, n²5, avril 2007, pp. 43-48.

39. L' ' Objective Force » peut être considérée comme la mise en forme stratégique du « système des systèmes » et tend à englober et à enraciner les multiples évolutions « transformationnelles » de la manœuvre réseau-centrique (Future Combat System, Land Warrior, fonctions C4ISR) dans un socle commun.

40. C'est à ce titre qu'un courant, porté par l'analyste Ralph Peters, conteste le développement des ANL au motif qu'il conduirait à délégitimer l'emploi de la force de dissuasion brute. 
sur l'adversaire. Le côté qui gagnera est celui qui pourra réduire davantage l'effet de cette brume et de cette friction par comparaison à son adversaire ${ }^{41}$ ».

Le «système des systèmes ", cette fameuse «force objective », s’instruit comme une tentative de limitation maximale, voire un dépassement du hasard et de l'incertitude caractéristiques de la pensée clausewitzienne. La «vision », ce «système des systèmes » idéal et partant éminemment fantasmatique, est à entendre moins comme une eschatologie que comme un attracteur idéologique, une tension permanente de la stratégie techno-militaire vers son pouvoir/devoirêtre. C'est dans cette manœuvre de projection et d'attraction que la «transformation ", comme prophétie auto-référentielle et auto-réalisatrice, trouve son fondement. Ce constat n'en invalide en aucun cas la puissance visionnaire, ni même la pertinence, pour peu que l'appareil organisationnel qui le prend en charge en assume pleinement les réussites et les échecs, les forces et les faiblesses.

Alors que dans ses applications antimatériel (neutraliser les centres de gravité adverses), le non-létal se rattache à l'approche techno-centrée de la guerre en réseau, il apparaît, dans sa raison politique (le «contrôle des foules ») comme un produit direct de la « guerre de quatrième génération ». Cette théorie née du retour d'expérience de la guerre du Golfe, est fondée sur le constat que le processus croissant de médiatisation/judiciarisation des conflits, comme la prolifération d'entités criminelles ou terroristes transnationales, modifie en profondeur la nature interactionnelle de la guerre et des règles de l'action militaire. La non-létalité se propose comme une riposte conceptuelle à cette mutation stratégique posée par la $4 \mathrm{GW}$, le fameux " facteur $C N N 42$ ». La «re-présentation » médiatique de l'action militaire comme subversion pouvant jouer pour ou contre la force place alors la guerre psychologique et les opérations informationnelles au centre de la stratégie post-moderne. Cette interaction cognitive du média dans le conflit est fondamentale pour appréhender le régime sociologique de la transformation de l'action militaire.

L'ANL est intervenue dans ce contexte comme une réponse pragmatique à ce défi juridico-médiatique posé à la force par la présence permanente et fluctuante des populations civiles dans les zones de guerre. C'est toute l'ambiguïté du concept que de prétendre se conformer aux impératifs, propres à la " guerre juste ", de proportionnalité et de discrimination entre combattants et non-combattants, et, dans le même temps, en prenant à partie la notion juridique de « légitime défense ", de légitimer l'extension policière du champ de la coercition militaire aux populations non directement combattantes. On n'est

41. Owens W.A., “The emerging system of systems”, National Defense University Strategic Forum, n63, février 1996.

42 . Humair D., Perron C., «Les armes non létales », Annuaire français de relations internationales, vol. 6, 2005. 
plus confronté dès lors à une guerre «totale », ordonnée par le principe de destruction indiscriminée des civils, mais à une forme réticulaire de guerre "globale », comme l'incarne aujourd'hui le conflit irakien, régi par un principe de paralysie discriminante, avec lequel c'est la frontière conceptuelle même entre combattants et non-combattants qui tend à disparaître.

Le non-létal, indissolublement lié au "continuum de force ", s'inscrit donc dans cette globalisation stratégique du phénomène "guerre ». Conséquence majeure, la mise sur pied de ce continuum exclut ou abolit de fait, dans sa définition même, la distinction symbolique, coutumière dans les armées européennes, entre « sécurité intérieure » et « sécurité extérieure ». Le concept d'ANL - parce qu'il fusionne en une seule pratique sécuritaire les deux aspects, policier et militaire, du contrôle du corps et des populations constitue dans cette approche le point d'achoppement et d'hybridation entre " contrôle des foules » et "maintien de l'ordre », entre «coercition de force » et «maitrise de la violence ». Il se projette donc comme un véritable point de rupture dans l'histoire de la culture tactique des forces terrestres, ce qui explique sans doute la réticence de certains états-majors européens à en faire un concept opérationnel autonome.

\section{L'arme non létale dans la «manœuvre génétique »}

Siégeant au cœur de la stratégie des moyens, la «manœuvre génétique » peut être perçue comme le processus de gestation, d'invention et de mise en œuvre de l'armement. Si l'on s'en réfère au lieutenant-colonel Becam ${ }^{43}$,

« la possibilité et la nécessité de manœuvrer dans le domaine de la génération des forces sont apparues lorsque l'arbre généalogique des armements s'est mis à pousser, à s'étendre et à se diversifier au point que d'une part on a pu choisir entre plusieurs formules ou systèmes d'armes alors que, d'autre part, on devenait incapable de fabriquer en nombre suffisant toutes les armes que la science et la technique permettraient de réaliser ».

La notion de «guerre technologique » mise en valeur par Possony et Pournelle ${ }^{44}$ reprend l'idée d'une autonomisation croissante de la manœuvre technique au cœur de la stratégie qui tendrait à supplanter ou recouvrir à terme la finalité politique de la guerre. Comme le souligne Joseph Henrotin ${ }^{45}$,

43. Lieutenant-colonel Becam, «La manœuvre génétique ", Forces aériennes françaises, n¹52, 1959.6, 2005.

44 . Possony S., Pournelle J., The Strategy of Technology: Winning the Decisive War, New-York, The Dunellen Company Inc., 1970. Disponible en ligne :

http://www.jerrypournelle.com/sot/sot_intro.htm

45 . Henrotin J., «De quelques fondements de la culture technologique américaine », Cabiers $d u$ RMES, vol. 2, $\mathrm{n}^{\circ} 2$, hiver 2005, pp. 25-45. Pour une étude détaillée du concept de «stratégie 
la manœuvre techno-militaire n'est plus alors « uniquement conçue comme moyen d'une fin politique mais devient bel et bien une stratégie en soi», elle procède d'une essentialisation de la technique propre à la RAM. La « manœuvre génétique » se définit dans cette optique comme un rapport complexe entre la culture stratégique et la culture technologique dans l'interfaçage entre les énoncés politico-militaires - stratégiques - et les phases successives de conception/production de l'armement.

Une brève étude du champ techno-militaire des armes à énergie dirigée fournit un exemple probant de cette interaction entre une projection stratégique/tactique (la non-létalité dans le continuum de force) et une technologie de pointe (l'énergie électromagnétique) et l'émergence d'un concept prospectif (l'armement « rhéostatique ») qui peut en résulter.

\section{L'énergie dirigée dans la "stratégie génétique "}

En avril 2005, Raytheon s'est vu attribuer par le Pentagone 7,5 millions de dollars pour finaliser la mise en service expérimentale d'un prototype de canon-àondes antipersonnel. Cette décision officialisait définitivement les recherches menées depuis le début des années 1990 sur une arme antipersonnel capable de protéger activement l'accès des zones sensibles et de briser les attroupements d'émeutiers. La déclassification en 2001 des recherches du laboratoire de Los Alamos sur les ondes électromagnétiques a permis de prendre connaissance de l'avancée des travaux exploratoires sur ces systèmes d'armes de nouvelle génération.

Ces armes à énergie dirigée (AED) représentent à l'heure actuelle l'un des domaines les plus prometteurs de la prospective de l'armement de haute technologie et témoignent de la volonté du Pentagone de maximaliser le potentiel de l'énergie dirigée pour renverser les schémas traditionnels de l'asymétrie. Depuis 1995, plus de 51 millions de dollars ont été assignés, entre autres, aux études sur les micro-ondes du laboratoire de l'Air Force à Kirtland. Ces programmes ont abouti en 200646 à la mise en service de deux principaux dispositifs à énergie dirigée antipersonnel, l'un à micro-ondes - l'Active Denial System (ADS) -, l'autre acoustique - le Long Range Acoustic Device (LRAD) ${ }^{47}$. Ces systèmes

génétique » voir: Henrotin J., La Stratégie génétique dans la stratégie des moyens, Paris, Institut de stratégie comparée, coll. "Les stratégiques », 2004.

46. Jontz S., "Troops in Iraq to get combined lethal/nonlethal weapons system", Stars and Stripes, éd. européenne, 14 septembre 2004.

47. Le Long Range Acoustic Device est un système d'hyperfréquence fondé sur une technique de génération de sons développée par la firme American Technology Corporation qui consiste à produire deux ultrasons de fréquences légèrement différentes : le signal haute fréquence se dissipera rapidement dans l'atmosphère, tandis que le signal basse fréquence va persister et peut être dirigé sur un groupe d'individus, entraînant nausées, malaises intestinaux, troubles de la vision et de l'ouie. Une variante portative est à l'étude, capable de tirer des «balles sonores » de forte intensité. Le LRAD a connu un premier emploi concluant en repoussant l'abordage du paquebot Seabourn Spirit par des pirates, au large des côtes somaliennes, en novembre 2005. 
sont actuellement déployés en Irak et font l'objet d'expérimentations actives sur la population civile. Ils ont notamment été testés dans le centre de Bagdad, dans la région de Falloujah et à Camp Bucca. On peut également mentionner, puisqu'il est en cours de finalisation industrielle, l'existence du Pulsed Energy Projectile (PEP) ${ }^{48}$, laser à deutérium fluoré développé par Mission Research Corp, dont les phases de test devraient débuter avant 2010. Ce développement augure la naissance d'une génération d'armes potentiellement révolutionnaires parce que susceptibles, comme l'ADS, d'investir la globalité du spectre opérationnel. On remarquera que tous ces dispositifs possèdent des versants létaux dans la gamme antimatériel : l'Advanced Tactical Laser pour l'ADS, le Pulsed Impulsive Kill Laser (PIKL) pour le PEP. Naturellement, l'ADS comme le LRAD connaissent aussi des versions civiles : le Silent Guardian ${ }^{49}$ pour l'ADS, le Mosquito de la firme britannique Compound Security Systems pour le LRAD, peuvent être cités comme exemplaires de cette dualité civile-militaire qui siège au cœur du concept de non-létalité.

\section{Combiner fonctions létale et non létale : vers l'armement "rhéostatique »}

La finalité tacite et critique d'un tel développement est de concevoir et de fabriquer, en se fondant sur les impératifs de graduabilité de la réponse armée et de réversibilité du mode tactique, un système combinant et modulant fonctions létale et non létale. Le but était, dès le début des années 1990, de « développer des armes à effets réglables, variant fonctions non létale et létale 50 ». C'est en 1998, dans un document intitulé Broad Area Announcement for NonLethal Technologies, qui établissait un catalogue prospectif des fonctions non létales, que la JNLWD énonce officiellement ce besoin capacitaire d' "un système d'arme unique dont les effets puissent couvrir l'ensemble du spectre d'intensité (du non-létal au létal) 51 ». La terminologie américaine emploie le terme d'armement «rhéostatique 52 ». Tout l'enjeu est de gérer le plus efficacement et le plus simplement possible le changement de posture, la « rétroaction » de la force brute à la force non létale et réciproquement. Le Joint Concept de 1998 reprend explicitement ce mot d'ordre :

«Pour que les ANL réalisent leur vrai potentiel, elles doivent être en mesure de produire plusieurs variétés d'effets. Cette caractéristi-

48. Voilà la définition prospective qu'en donne le National Research Council : «Le PEP utiliserait un laser au fluorure de deutérium à impulsions conçu pour produire un plasma ionisé à la surface de la cible visée. Le plasma entraînerait à son tour une onde de pression ultrasonique qui passerait dans le corps et stimulerait les nerfs cutanés afin de provoquer une douleur et une paralysie temporaire».

49. Voir http://www.raytheon.com/products/silent_guardian

50. Tapscott M., Atwal K., "New weapons that win without killing on DOD's horizon”, Defense Electronics, pp. 41-46, février 1993. Notre traduction.

51. Marine Corps System Command, Research and Development for Non-Lethal Technologies, SOL BAA-98-R-0016, Commerce Business Daily, 12 mai 1998.

52 . Par référence au rhéostat, résistance variable destinée à régler la puissance dans un circuit. 
que - une fonction "rhéostatique" ou "réglable” - permettra aux commandants d'accroître ou de décroître le degré d'influence nécessaire à l'obtention de l'obéissance. Une capacité rhéostatique fournit une palette d'effets nécessaires à l'investissement total du continuum de force 53 ».

Révolutionnaire, l'énergie dirigée l'est à trois titres : une telle technologie combine virtualités antipersonnel et antimatériel ; peut fonctionner comme arme individuelle ou arme à effet de zone ; enfin et surtout, peut graduer/permuter fonction létale et fonction non létale. L'Active Denial System, système phare promu par le JNLWD, correspond en tous points à ce modèle génétique et mérite une analyse particulière.

L'ADS repose sur une technologie apparentée à celle de l'Advance Tactical Laser, évoquée précédemment, mais contrairement à l'ATL, qui est un laser à micro-ondes de haute puissance, toujours en phase d'étude, censé conférer à terme une précision létale sans précédent aux forces aériennes, l'ADS est un canon à ondes millimétriques pulsées de basse puissance et une technologie pleinement opérationnelle. Baptisé « projet Sheriff », ce système tactique voué à l'engagement rapproché se compose d'un émetteur de moyenne portée 54 incrusté dans une antenne orientable. Il peut être monté selon les besoins sur le toit d'un poste de garde comme arme à effet de zone ou sur des véhicules type Humvee, Stryker ou Light Armored, comme arme anti-émeute. Quant à la mise en œuvre physique du canon, elle est relativement simple : le rayon émet sur une fréquence de 95-100 GHz, insuffisante pour pénétrer le corps ${ }^{55}$, mais assez forte pour réagir avec les terminaisons nerveuses du derme superficiel et chauffer les molécules d'eau des couches sous-cutanées, provoquant au bout de cinq secondes une sensation de brûlure si intense que le réflexe naturel de l'individu est de prendre immédiatement la fuite. Le rayon émis n'est pas ionisant - non radioactif - et par conséquent ne comporte pas de danger carcinogène. C'est donc, en tous les cas sur le papier, une arme "propre », sans effet secondaire avéré pour le moment ${ }^{56}$. Outre le fait qu'il puisse être employé comme un moyen de conditionnement et de torture particulièrement efficace, le principal non-dit de ce système réside dans son potentiel létal. En effet, s'il est pour l'heure conçu et destiné à un usage non létal, il est techniquement tout à fait envisageable d'accroître la pulsation du rayon pour en faire une arme mortelle, «rhéostatique ». Une autre incertitude concerne les conséquences pour le cerveau d'une exposition répétée au

53. Joint Concept for Non-Lethal Weapons, Commandant of the Marine Corps, 5 janvier 1998. Notre traduction.

54. Champ effectif de 500 à 750 mètres maximum.

55 . La pénétration est de l'ordre de $1 / 64$ inch, soit environ $0,4 \mathrm{~mm}$, pour un pic de température pouvant atteindre $54^{\circ} \mathrm{C}$.

56. D’après le site de la JNLWD, seules deux brûlures au second degré ont été constatées en douze ans de tests sur la base de Kirtland. 
rayon. Bien que les chercheurs militaires démentent les risques de pathologies encéphaliques, le facteur de la durée d'exposition reste central.

Ce système a-t-il un avenir tactique ? Sa mise en service officielle est pour l'heure retardée ${ }^{57}$, notamment en raison des retombées médiatiques que l'emploi d'une telle arme pourrait susciter dans la gestion post-conflit et ce, en dépit des doléances des commandants de terrain, échaudés par les expériences désastreuses de contrôle des foules vécues par l'armée américaine. Les massacres de manifestants à Mossoul et Falloujah ${ }^{58}$, quelques semaines seulement après l'invasion, ont joué un rôle prépondérant dans l'accélération des programmes de développement des ANL de seconde génération. Il est donc peu probable que l'ADS en vienne à dépérir par simple pudeur éthique (il pourra à cet égard compter sur la légitimation médiatico-politique dont a fait l'objet le Taser), surtout quand on constate l'ampleur du marché économique, militaire et civil qui existe pour ce type de solutions. De nombreuses déclinaisons du système sont d'ores et déjà envisagées : versions aéroportées, versions portatives en arme de poing ou, comme l'a proposé la firme Metal Storm, une version montée sur un drone de combat terrestre, même si pour l'heure cette application reste purement spéculative. Autres possibilités, il serait utilisable dans des versions automatisées pour interdire l'abord des sites nucléaires ou des ambassades, ou encore l'accès à d'autres zones énergétiques, comme les points émergés des pipelines, pour contrer l'économie criminelle liée à la rente pétrolière en Afrique subsaharienne. A l'instar du LRAD, il pourrait également équiper les navires marchands, tankers, et autres bâtiments susceptibles d'être la proie de pirates ou de terroristes, même si la portée restreinte du canon fragilise cette dernière hypothèse.

Reste que le concept d'armement «graduable » ou «rhéostatique » représente à l'évidence une rupture épistémique dans l'histoire de la "politique " de conception et de production des moyens de la guerre. Surtout, en permettant d'imaginer un système d'armes trans-spectre (full spectrum), pouvant être employé dans des opérations de basse, moyenne et haute intensité, il fournit un fondement technoscientifique concret aux arguments d'Alexander sur la stratégie de la «camisole », fondée sur une capacité de ciblage optimale et la mise en place d'un continuum de l'emploi de la force pouvant mobiliser une gamme variée d'armes non létales antipersonnel et antimatériel.

Au terme de cette analyse, l'arme non létale apparaît bien comme un concept déterminant de la conflictualité post-moderne. Parce qu'elle place au

57. Voir Lardner R., "Commanders in Iraq request energy beam weapon: concern it could be seen as torture", San Diego Union Tribune, 29 août 2007.

58 . Le 15 avril 2003 , quelques semaines après l'invasion de l'Irak, une opération de contrôle des foules dans Mossoul tourne au massacre, sept civils sont tués. Le 28 avril 2003, le même scénario se répète à Falloudjah. Le bilan sera de dix-huit morts et soixante-dix-huit blessés. 
centre des pratiques de la recherche techno-militaire la bio-ingénierie du corps humain, la théorie de la non-létalité introduit dans l'action militaire le règne d'une biostratégie, d'une techno-stratégie du vivant, non plus déterminée par la destruction du corps ennemi et le primat ancestral, dans la pensée de la guerre, du pouvoir de mort, mais par l'exercice d'un contrôle permanent par et sur les corps. L'interrogation éthique sur l'armement non létal, son degré d'acceptabilité morale, est bien sûr un élément crucial du débat, mais elle ne peut guère nous aider à apercevoir l'horizon définitoire du concept, celui d'un enracinement bio-technologique de la manœuvre politique et stratégique de gestion et de contrôle des corps et des populations. C'est sans doute dans cette transition, en tant que principe de la guerre, de la « destruction » à la «paralysie », que se découvre l'enjeu proprement biopolitique de la théorie de la non-létalité, dans ce passage du corps ennemi « anéanti » au corps ennemi "réifié », et bientôt, par le biais des nanotechnologies, « investi » bioniquement. La présentation des ANL comme une inflexion humanitaire de l'action militaire fonctionne dans ce cadre comme une structure de méconnaissance, derrière laquelle c'est en réalité une évolution, immanente à la stratégie génétique, vers un posthumanisme, qui s'opère. Cette évolution correspond-t-elle aux nécessités stratégiques de la contre-insurrection en Irak et plus généralement aux impératifs futurs de la guerre dite « asymétrique » ? Quel impact aura-t-elle sur la culture tactique des armées occidentales ? Ces enjeux, critiques pour l'avenir des forces terrestres et l'éthique des armes, sont désormais posés historiquement. 